\title{
Effect of Electron Beam Irradiation and Heating on the Structural Stability of Sulphide-Filled Carbon Nanotubes
}

\author{
P. M. F. J. Costa***, U.K. Gautam********, Y. Bando**** and D. Golberg**** \\ * CICECO, Department of Ceramics and Glass Engineering, University of Aveiro, 3810-193 Aveiro, \\ Portugal \\ ** IFW-Dresden, 01069 Dresden, Germany \\ *** New Chemistry Unit, JNCASR, 506-064 Bangalore, India \\ **** MANA, National Institute for Materials Science, 305-0044 Ibaraki, Japan
}

In recent years there has been an increased interest in characterisation methods capable of evaluating the response of nanostructured materials such as quantum dots and nanotubes to externally applied stimuli. However, due to issues concerning sample homogeneity and non-uniform orientation, the study of collective responses can lead to ambiguous results. Therefore, it is crucial to perform a complementary evaluation at the single nanostructure level which requires the use of sophisticated analytical instrumentation. In addition to high spatial resolution, the equipment employed must permit sample manipulation and real-time imaging of the in situ stimulus response. Transmission electron microscopes (TEM) observe all these requirements. Furthermore, novel developments in sample holder design and manufacturing have opened a window to an unprecedented range of new in situ experiments. As a result, varied reports are increasingly populating the in situ TEM literature examining such different matters as temperature-related structural phase changes of nanoparticles [1] or dopant diffusion in nanoscaled batteries [2].

One major criticism to in situ TEM studies is the influence of the imaging electron beam on the behaviour of the stimulated material. At several tenths (or hundreds) of electron-volts of kinetic energy, the charged particles can transfer momentum and energy to the sample under observation. Indeed, if the particles are densely arranged at the object plane, pronounced changes in the structure and chemistry of the material may take place thus invalidating the in situ observations. It is thus imperative to decouple, or at least understand, the effect of the electron beam from the intrinsic results derived from the stimulus application.

Recently, we have been examining the response of Ga-doped $\mathrm{ZnS}$ encapsulated in carbon nanotubes (a.k.a. $\mathrm{Zn}_{0.92} \mathrm{Ga}_{0.08} \mathrm{~S} @ \mathrm{CNT}$ ) to a number of external stimuli using in situ TEM methods [3-5]. Amongst our investigations was the response of $\mathrm{Zn}_{0.92} \mathrm{Ga}_{0.08} \mathrm{~S} @ \mathrm{CNT}$ to a high density of electrical current using a two-terminal probing sample holder $[5,6]$. Real-time imaging of structural changes in the sulphide core suggested the presence of a prominent Joule heating effect. In turn, this observation raised questions on how the electron beam would influence these changes both at room and high temperatures.

We have analysed how the electron beam and heat interact with the $\mathrm{Zn}_{0.92} \mathrm{Ga}_{0.08} \mathrm{~S} @ \mathrm{CNT}$ (Figure 1a). Initially, the core-shell system was exposed, at room temperature, to different electron doses using a converged $300 \mathrm{keV}$ electron beam. Noticeable structural rearrangements followed which ultimately lead to complete drilling of the nanotube (Figure 1b). Such a localised effect was in strong contrast to the response of the system at high temperature $(773 \mathrm{~K})$. At similar dosage, the core sulphide was quickly depleted, well before full drilling of the nanotube had taken place (Figure 1c). Furthermore, the interaction volume of the beam was considerably extended.

We are now extending this work to understand better the mechanisms responsible for this differentiation. Nonetheless, it is clear that temperature and electron beam irradiation can be used in tandem to obtain varied responses from $\mathrm{Zn}_{0.92} \mathrm{Ga}_{0.08} \mathrm{~S} @ \mathrm{CNT}$. 


\section{References}

1. Young N.P. et al., , «Transformations of gold nanoparticles investigated using variable temperature high-resolution transmission electron microscopy», Ultramicroscopy 110: 506, 2010

2. Huang J.Y. et al., «In Situ Observation of the Electrochemical Lithiation of a Single SnO2 Nanowire Electrode», Science 330: 1515, 2010

3. Costa P.M.F.J. et al., «Effect of crystalline filling on the mechanical response of carbon nanotubes», Carbon 47: 541, 2009

4. Costa P.M.F.J. et al., «Imaging the Oxidation of ZnS Encapsulated in Carbon Nanotubes», Chem. Eur. J. 16: 11809, 2010

5. Costa P.M.F.J. et al., «The electrical delivery of a sublimable II-VI compound by vapor transport in carbon nanotubes», Carbon 49: 3747, 2011

6. Costa P.M.F.J. et al., «Direct imaging of Joule heating dynamics and temperature profiling inside a carbon nanotube interconnect», Nature Comms. 2: 421, 2011
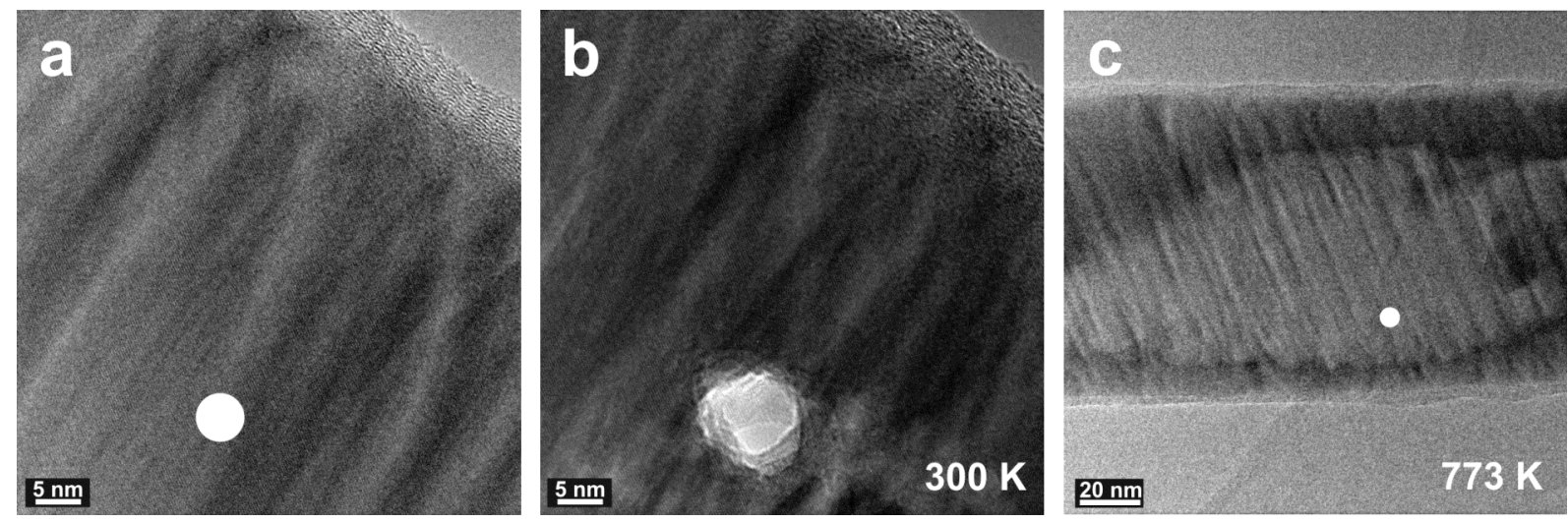

Figure 1. (a) Initial configuration of a Zn0.92Ga0.08S@CNT. (b) The structure in (a) after 5 minutes of exposure to the electron beam at room temperature. (c) A Zn0.92Ga0.08S@CNT after 2 minutes of exposure to the converged electron beam at high temperature. A large volume of the filling has been sublimed. The white circles in (a) and (c) indicate approximately the area sampled by the converged electron beam. 